\title{
BOLO TIPO BROWNIE GLÚTEN FREE OBTIDO A BASE DE FARINHA DE BATATA-DOCE (IPOMOEA BATATAS)
}

\author{
M.M. B. de OLIVEIRA ${ }^{1}$, M.M.A. MIRANDA ${ }^{2}$, S.C. de MELO FILHO ${ }^{3}$, \\ M.A.S.VASCONCELOS ${ }^{4}$ \\ 1,2 Universidade Federal de Pernambuco, Centro de Ciências da Saúde, Departamento de \\ Ciências Farmacêuticas \\ ${ }^{3,4}$ Universidade Federal de Pernambuco, Centro de Ciências da Saúde, Departamento de \\ Nutrição
}

E-mail para contato: mmbonutricao@gmail.com

\begin{abstract}
RESUMO - Este trabalho teve como objetivo obter e caracterizar a farinha de batatadoce (FBD), bem como aplicá-la em produto alimentício bolo tipo Brownie. A pesquisa consistiu na obtenção da farinha por desidratação sob circulação de ar para a elaboração de bolo tipo brownie glúten free. Também foi determinada a composição centesimal obtendo-se as frações centesimais de umidade, cinzas, gordura total, proteína total, carboidratos por diferença e valor calórico total. A farinha experimental foi aplicada na elaboração de bolos tipo Brownies de chocolate com substituição total e parcial da farinha de trigo. Como resultados, verificou-se que a farinha de batatadoce se caracteriza como fonte potencial de amido para substituição ao trigo e possui amido mais resistente ao processamento, além de apresentar baixo teor de lipídeos e boa fonte de proteinas quando comparadas a fontes vegetais tradicionais e se mostrou um alimento com elevado valor nutricional. Dos bolos tipo Brownie, com a crescente substituição da farinha de trigo pela FBD verificou-se perda de altura da massa, fato que não interferiu na textura, odor e sabor do produto final. Assim a FBD mostrou-se como uma excelente alternativa para pacientes celíacos ou pessoas que optem por um produto glúten free.
\end{abstract}

Palavras-chave: Batata-doce. Farinha de batata-doce. Brownie.

ABSTRACT - This work aimed to obtain and characterize sweet potato flour (FBD) as well as apply it in brownie type cake food product. The research consisted in obtaining the flour by dehydration under air circulation for the preparation of brownie type gluten free cake. It was also determined the centesimal composition obtaining the centesimal fractions of moisture, ashes, total fat, total protein, carbohydrates by difference and total caloric value. The experimental flour was applied in the elaboration of chocolate brownies type cakes with total and partial substitution of wheat flour. As results, it was verified that sweet potato flour is a potential source of starch for wheat substitution and has a starch that is more resistant to processing, besides presenting low lipid content and good protein source when compared to traditional vegetable sources and showed to be a food with high nutritional value. From the Brownie type cakes, with the increasing substitution of the wheat flour by the FBD, loss of height of the pasta was verified, a fact that did not interfere 
in the texture, odor and flavor of the final product. Thus FBD has proven to be an excellent alternative for celiac patients or people who opt for a gluten free product.

Keywords: Sweet potato. Sweet potato flour. Brownie.

\section{INTRODUÇÃO}

A batata-doce é uma hortaliça de raízes tuberosas, adaptada a diversas condições de solo e de clima, alta tolerância à seca, baixo custo de produção e cultivada tanto nos trópicos como nas regiões temperadas do planeta (SOARES et al., 2014). Pode ser empregada na alimentação humana e animal e como matéria-prima nas indústrias de alimentos, tecidos, papel, cosméticos, preparação de adesivos e álcool carburante (CARDOSO et al., 2005).

Considerada a quarta hortaliça mais consumida no Brasil, a batata-doce se constitui numa fonte de energia e nutrientes na dieta alimentar humana (OLIVEIRA et al., 2013). As raízes apresentam teor de carboidratos variando entre $25 \%$ a $30 \%$, dos quais $98 \%$ são facilmente digestíveis. Também são excelentes fontes de carotenóides, vitaminas do complexo B, potássio, ferro e cálcio (SILVA, 2010).

Para a viabilização da introdução de uma nova matéria-prima nas indústrias produtoras de amido é preciso considerar a parte agrícola da cultura, a composição físicoquímica da porção a ser processada e os resíduos gerados. Sob o ponto de vista tecnológico, a presença de outros componentes também pode ser considerada (MASCARENHAS; RESENDE, 2003).

Diante disso, na indústria alimentícia o amido é utilizado em função da viscosidade, poder gelificante, adesão, tendência a retrogradação, entre outras propriedades que são influenciadas pela proporção amilose e amilopectina, teor de proteína e gordura além da estrutura, forma e tamanho dos grânulos (DAIUTO et al., 2002). A substituição de farinha de trigo por outros tipos de farinhas é uma forma de se melhorar a alimentação para que se tenha uma vida mais saudável, além de ser uma excelente opção para intolerantes ou alérgicos a componentes do trigo como, por exemplo, o glúten (FERREIRA, 2003).

Nesse contexto, a produção de farinha de batata-doce apresenta grande versatilidade para a indústria de alimentos, principalmente em produtos de panificação, produtos dietéticos e alimentos infantis, por ser rica fonte de amido e sais minerais. Sem afetar a qualidade do produto final e sem precisar fazer grandes modificações, a farinha de batata-doce já é usada para substituir parcialmente a farinha de trigo na fabricação de massas alimentícias frescas e secas (CARVALHO et al., 2005).

O presente trabalho visou à obtenção de farinha de batata-doce, como uma matéria-prima para obtenção de fonte amilácea, para a aplicação na substituição parcial e total da farinha de trigo comercial em produtos alimentícios como o Brownie com potencialidade para atingir mercado glúten free.

\section{MATERIAIS E MÉTODOS}

\subsection{OBTENÇÃO DA FARINHA EXPERIMENTAL DE BATATA-DOCE (FBD)}

$\mathrm{O}$ procedimento para obtenção da farinha de batata-doce seguiu a técnica de desidratação em estufas com circulação de ar, seguindo o descrito por Souza et al (2012), 
com modificações. Após seleção, sanitização, descascamento e corte as lâminas seguiram para o desidratador previamente aquecido a $65^{\circ} \mathrm{C}$, até atingirem completa secagem, estágio em que se apresentem com aspecto quebradiço (aproximadamente $8 \mathrm{~h}$ ).

Após a etapa de desidratação a farinha foi obtida por trituração em liquidificador, passando por peneiragem em tamis (um milimetro de abertura nas peneiras/ 16 mesh) até obter-se a farinha com granulometria semelhante à farinha de trigo. A farinha foi acondicionada em recipientes esterilizados, secos e com fechamento hermético.

\subsection{COMPOSIÇÃO CENTESIMAL}

A composição centesimal da farinha FBD foi determinada através da aplicação de metodologias com modificações segundo Instituto Adolfo Lutz (2008), obtendo-se as frações centesimais de umidade e sólidos totais, pelo método gravimétrico a peso constante (estufa a $105^{\circ} \mathrm{C}$ ), cinzas totais por gravimetria com incineração a $550^{\circ} \mathrm{C}$, gordura total por extração a quente pelo método de Soxhlet, proteína total pelo método de Kjeldahl (fator de correção 6,5) e carboidratos por diferença.

\subsection{APLICAÇÃO DA FARINHA DE BATATA-DOCE EM PRODUTO ALIMENTÍCIO}

A farinha de batata-doce foi testada na formulação de Brownie de chocolate, sendo utilizada em substituição parcial e total da farinha de trigo. Foram produzidos três Brownies, um padrão (utilizando a farinha de trigo); com substituição parcial de $50 \%$ da farinha de trigo por FBD (B1); e com substituição total de farinha de trigo pela FBD (B2).

$\mathrm{Na}$ formulação dos Brownies a farinha de trigo e a farinha de batata-doce foram os únicos ingredientes que variaram. Os ingredientes e suas proporções estão descritos na Tabela 1.

Tabela 1. Formulação para elaboração dos Brownies de chocolate com farinha de trigo e com substituição de 50\% de FBD e 100\% de FBD.

\begin{tabular}{cccc}
\hline Ingredientes & Padrão & $\begin{array}{c}\text { B1 (50\% de } \\
\text { FBD) }\end{array}$ & $\begin{array}{c}\text { B2 (100\% de } \\
\text { FBD) }\end{array}$ \\
\hline Farinha de Trigo & $150 \mathrm{~g}$ & $75 \mathrm{~g}$ & - \\
Farinha de batata- & - & $75 \mathrm{~g}$ & $150 \mathrm{~g}$ \\
doce & $200 \mathrm{~g}$ & $200 \mathrm{~g}$ & $200 \mathrm{~g}$ \\
Açúcar & $178 \mathrm{~g}$ & $185 \mathrm{~g}$ & $183 \mathrm{~g}$ \\
Ovo & $150 \mathrm{~g}$ & $150 \mathrm{~g}$ & $150 \mathrm{~g}$ \\
Margarina & $1 \mathrm{~g}$ & $1 \mathrm{~g}$ & $1 \mathrm{~g}$ \\
Essência de & & & $1 \mathrm{~g}$ \\
baunilha & $1 \mathrm{~g}$ & $\mathrm{~g}$ & $1 \mathrm{~g}$ \\
$\begin{array}{c}\text { Bicarbonato de } \\
\text { sódio }\end{array}$ & $200 \mathrm{~g}(1 / 2$ ao leite e $1 / 2$ & $200 \mathrm{~g}(1 / 2$ ao leite e & $200 \mathrm{~g}(1 / 2$ ao leite e \\
Chocolate em barra & meio amargo $)$ & $1 / 2$ meio amargo $)$ & $1 / 2$ meio amargo $)$ \\
Chocolate em pó & $18 \mathrm{~g}$ & $18 \mathrm{~g}$ & $18 \mathrm{~g}$ \\
\hline
\end{tabular}

A massa de Brownie foi obtida em duas etapas. Inicialmente, o chocolate em barra foi derretido em banho-maria juntamente com a margarina e em seguida foi adicionada a essência de baunilha, misturando-se até obter aparência homogênea ficando reservada para segunda etapa. Em outro recipiente misturou-se os ovos com o açúcar e foi acrescentada a mistura com chocolate reservada. Após mexer e obter a massa foi 
adicionada a farinha, o chocolate em pó e o bicarbonato, misturando-se tudo, obtendo assim ao final a massa do Brownie.

A massa foi colocada em formas assadeiras de $21 \times 32 \mathrm{~cm}^{2}$ forradas com papel manteiga e levadas ao forno pré-aquecido a $200^{\circ} \mathrm{C}$ por cerca de 40 minutos. Para as massas experimentais, houve apenas a substituição da farinha de trigo pela FBD em 50\% e $100 \%$.

Foi realizada a caracterização física dos Brownies sob os seguintes parâmetros: peso pré e pós-cocção $(\mathrm{g})$, volume $\left(\mathrm{cm}^{3}\right)$, volume específico $\left(\mathrm{cm}^{3} / \mathrm{g}\right)$, altura $(\mathrm{cm})$, fator térmico e perdas na cocção (\%). As formas com a massa dos Brownies foram pesadas em balança digital antes e após cocção para realizar um comparativo de peso dos Brownies experimentais com o Brownie padrão.

Para a obtenção do volume foi marcada nas formas com caneta apropriada a altura alcançada pelo Brownie cozido, as quais foram preenchidas com água, até a marcação. Em seguida, despejou-se a água em proveta graduada (GUIMARÃES, FREITAS e SILVA, 2010) e o volume específico foi calculado pela razão volume/peso (BASSAN, FERREIRA e ESCOUTO, 2009). A medida da altura foi realizada com uso régua milimetrada (MAZIERO et al., 2009). Essa medida foi realizada para avaliar a expansão de cada Brownie experimental produzido para fazer uma comparação com o Brownie padrão.

O fator térmico foi determinado pela razão entre o peso dos produtos antes e após a cocção (GUIMARÃES, FREITAS e SILVA, 2010).

Para obtenção das perdas na cocção foi realizado a subtração do peso pré-cocção pelo peso pós-cocção e expressas em forma de percentagem (BASSAN, FERREIRA e ESCOUTO, 2009).

\section{RESULTADOS E DISCUSSÃO}

\subsection{COMPOSIÇÃO CENTESIMAL}

A composição centesimal de um alimento exprime de forma básica o valor nutritivo ou valor calórico, bem como a proporção de componentes em que aparecem, em 100g de produto (porção comestível do alimento) (MORETO et al., 2002).

Tabela 2. Composição centesimal (g/100g) e valor calórico total (VCT) (Kcal/100g) da farinha de batata-doce e de farinha de trigo.

\begin{tabular}{ccc}
\hline Componentes & $\begin{array}{c}\text { Farinha de batata- } \\
\text { doce }\end{array}$ & Farinha de trigo* \\
\hline Umidade e substâncias & 8,23 & 13 \\
voláteis & 12,35 & 9,8 \\
Proteínas Totais & 2,69 & 1,4 \\
Lipídeos Totais & 2,54 & 0,8 \\
Cinzas & 74,19 & 75,1 \\
Carboidratos & 370,37 & 360 \\
Calorias & & \\
\hline
\end{tabular}

*Fonte: (TACO, 2011)

Diante da comparação da farinha de batata-doce com a farinha de trigo, verificase que a FBD apresentou menor umidade mediante tempo e temperaturas utilizados, podendo então apresentar uma estabilidade maior ao crescimento microbiano, que pode inferir no tempo de vida útil de prateleira. 
A quantidade de proteínas encontrada foi superior para a FBD apresentando um valor muito próximo ao da quinoa $(12 \mathrm{~g} \%)$ e da soja $(12,5 \mathrm{~g} \%)$, que são consideradas ótimas fontes de proteína vegetal (KONISHI et al., 2004).

A quantidade de lipídeos da FBD foi superior em relação à farinha de trigo, mas ainda apresenta uma quantidade bem menor do que a farinha aveia $(3,5 \mathrm{~g} \%)$ e a farinha láctea $(7,8 \mathrm{~g} \%)$ (TBCAUSP, 2008).

A determinação das cinzas é uma análise importante para verificar alterações na composição dos alimentos bem como seus minerais e nutrientes. $\mathrm{O}$ valor de cinzas foi relativamente maior para a $\mathrm{FBD}$, sendo de $2,54 \mathrm{~g}$, quando comparada à farinha de trigo $(0,8 \mathrm{~g})$, podendo ser indicativo de maior teor de minerais.

O valor para carboidratos foi muito próximo nas duas farinhas, como já esperado, pois ambas são compostas por uma quantidade elevada de amido, sendo que a FBD apresenta um menor índice glicêmico.

\subsection{BROWNIES DE CHOCOLATE: APLICAÇÃO DE SUBSTITUIÇÃO PARCIAL E TOTAL DA FARINHA DE TRIGO POR FBD}

A Tabela 3 apresenta os parâmetros que caracterizam a influencia da substituição da farinha de trigo por FBD no produto final: peso pré e pós-cocção $(\mathrm{g})$, volume $\left(\mathrm{cm}^{3}\right)$, volume específico $\left(\mathrm{cm}^{3} / \mathrm{g}\right)$, altura $(\mathrm{cm})$, fator térmico e perdas na cocção $(\%)$.

Esses dados foram utilizados para caracterização física do produto experimental, os Brownies de chocolate padrão produzidos com $100 \%$ de farinha de trigo (BP), $50 \%$ de farinha de trigo com 50\% de farinha de batata-doce (B1) e com $100 \%$ de farinha de batatadoce (B2).

Tabela 3. Parâmetros para caraterização física dos Brownies de chocolate padrão (BP) e experimental (B1 e B2).

\begin{tabular}{cccc}
\hline Parâmetros & BP & B1 & B2 \\
\hline Peso pré-coç̧ão & $1143 \mathrm{~g}$ & $1141 \mathrm{~g}$ & $1147 \mathrm{~g}$ \\
Peso pós-cocção & $1029 \mathrm{~g}$ & $1030 \mathrm{~g}$ & $1070 \mathrm{~g}$ \\
Tempo de coç̧ão & 40 minutos & 40 minutos & 35 minutos \\
Altura & $2,2 \mathrm{~cm}$ & $1,5 \mathrm{~cm}$ & $1,4 \mathrm{~cm}$ \\
Volume & $1840 \mathrm{~cm}^{3}$ & $1570 \mathrm{~cm}^{3}$ & $1470 \mathrm{~cm}^{3}$ \\
Volume específico & $1,78 \mathrm{~cm}^{3} / \mathrm{g}$ & $1,52 \mathrm{~cm}^{3} / \mathrm{g}$ & $1,37 \mathrm{~cm}^{3} / \mathrm{g}$ \\
Fator térmico & 1,11 & 1,11 & 1,07 \\
Perdas na cocção & $9,97 \%$ & $9,72 \%$ & $6,71 \%$ \\
\hline
\end{tabular}

Em relação ao peso pré e pós-cocção o Brownie que utilizou $100 \%$ de FBD foi o que apresentou a menor diferença desses parâmetros, obtendo então a menor perda pós cocção, enquanto o brownie com $100 \%$ de farinha de trigo obteve esse valor mais elevado e muito próximo do brownie elaborado com $50 \%$ da FBD o que infere maior perda de umidade no produto final. Observa-se também o mesmo valor de fator térmico para os dois primeiros casos e uma pequena redução para o último. O tempo de cocção para os Brownies BP e B1 foi o mesmo, 40 minutos, enquanto que para o Brownie B2 esse tempo reduziu 5 minutos.

As diferenças na altura dos Brownies reflete a ausência gradual de glúten na massa, pela substituição da farinha de trigo, componente importantíssimo para a manutenção do volume de produtos de pastelaria e panificação. Os Brownies com redução (B1) ou ausência (B2) de farinha de trigo tiveram um menor crescimento e um 
menor volume final devido a redução ou ausência do glúten. A importância do glúten nos processos de panificação está basicamente ligada a sua capacidade de dar extensibilidade e consistência à massa, além de reter o gás carbônico proveniente da fermentação, promovendo o aumento de volume desejado (CUNHA, RUFFI, NABESHIMA, 2009).

A substituição de farinha de trigo por outras farinhas pode provocar mudanças no comportamento estrutural da massa. As alterações ocorrem na característica da massa, no tempo de fermentação e na qualidade final do produto (PIERARSKI, 2009). Com isso há uma redução maior do volume quando se trata da massa do Brownie elaborado com diminuição ou ausência da farinha de trigo, bem como diminuição do volume específico.

Apesar da dificuldade em encontrar trabalhos com farinha de batata-doce, alguns autores puderam contribuir com a aplicação dessa farinha em algumas formulações como Araújo (2015) que produziu pães com adição de farinha de batata-doce nas concentrações de 10,20 e $30 \%$ e observou que é um produto tecnologicamente viável, pois os resultados da análise sensorial demonstraram uma satisfatória aceitação do produto quanto aos atributos de cor, aparência, odor e sabor. Freitas (2014) elaborou bolos com substituição de $100 \%$ da farinha de trigo pela farinha de batata-doce e obteve uma excelente aceitação em análise sensorial.

Já Simon (2014) utilizou farinha de arroz e farinha de trigo sarraceno como substitutos parciais da farinha de trigo, com adição de goma xantana, para elaboração de Brownie e obteve aceitação superior a $70 \%$ em um painel sensorial não celíaco e as formulações apresentaram resultados satisfatórios também para análises de textura.

Verificou-se influência positiva na textura, com característica de mais úmido com o aumento da presença de FBD nas formulações, mesmo o Brownie padrão (BP) tendo o crescimento da massa superior aos experimentais, enquanto que para os aspectos sensoriais de sabor e o odor todos ficaram muito semelhantes. Destaca-se apenas para a cor o B2, que apresentou uma intensidade maior que os demais.

\section{CONCLUSÃO}

A FBD obteve resultados físico-químicos compatíveis com a literatura, mostrando-se viável e com características que favorecem sua estabilidade.

O Brownie obtido com substituição parcial e total da farinha de trigo, não teve seu aspecto sensorial final comprometido, mantendo textura e umidade semelhante ao Brownie padrão.

Portanto, os resultados apresentados nesse trabalho indicam que o Brownie produzido com farinha de batata-doce apresenta-se como alternativa para pessoas que optam por um produto glúten free, além de ser um alimento com elevado valor nutricional.

\section{REFERÊNCIAS}

ARAÚJO, C. S. P. Processamento da batata-doce para utilização em produto de panificação. Trabalho de conclusão de curso, Paraíba, 2015.

BASSAN, J.C.; FERREIRA G.A.O.; ESCOUTO, L.F.S. Avaliação física sensorial de pão de forma doce elaborado com farinha de batata doce de polpa alaranjada e mistura de amidos nativos e modificados isento glúten. XIII Congresso Brasileiro de Mandioca, 2009. UNESP - São Paulo. 
BRASIL. Métodos físico-químicos para análise de alimentos. Ministério da Saúde. Agência Nacional de Vigilância Sanitária. 4 ed. Instituto Adolfo Lutz. Brasília: Ministério da Saúde, p.1018. 2008.

CARDOSO A.D.; VIANA A.E.S.; RAMOS P.A.S.; MATSUMOTO S.N.; AMARAL C.L.F.; SEDIYAMA T.; MORAIS O.M. 2005. Avaliação de clones de batata-doce em Vitória da Conquista. Horticultura Brasileira 23:911-914.

CARVAlHO, F. M.; SANTOS, A.; VIANA, A. E. S.; LOPES, S.C.; EGLER, P.G. Avaliação da atividade poluidora da manipueira na bacia do Rio Santa Rita em Vitória da Conquista, Bahia. In: CONGRESSO BRASILEIRO DE MANDIOCA, 11, 2005. Campo Grande, MS. Resumos. Campo Grande: 2005

CUNHA, R. K.; RUFFI, C. R.; NABESHIMA, E. H. Glúten: importância tecnológica, doença celíaca, legislação e métodos de quantificação, disponível em Doce Revista. 2015.

DAIUTO, E.R.; CEREDA, M.P.; CARVALHO, L.J.C.B. Características e propriedades do amido extraído de camadas do tecido da raiz de mandioca. Brazilian Journal Of Food Technology, v. 5, p. 217-223, 2002.

FERREIRA, R. A. Trigo: o alimento mais produzido no mundo. Nut. Brasil, São Paulo, v. 2, n. 1, p. 45-52, 2003.

FRANCO, C. M. L. et al. Propriedades do Amido. In: Culturas de Tuberosas Amiláceas Latino Americanas, Propriedades Gerais do Amido. Campinas, Fundação Cargill, 2001.

FREITAS, M. A. D. Elaboração e aceitabilidade de bolos enriquecidos com farinha de batata-doce. Trabalho de conclusão de curso, Paraíba, 2014.

GUIMARÃES, R. R.; FREITAS, M. C. J. de; SILVA, V. L. M. da. Bolos simples elaborados com farinha da entrecasca de melancia (Citrullus vulgaris, sobral): avaliação química, física e sensorial. Ciência e Tecnologia de Alimentos. Campinas, v. 30, n. 2, p. 354-363, 2010.

KONISHI, Y.; HIRANO, S.; TSUBOI, H.; WADA, M. Distribution of minerals in quinoa (Chenopodium quinoa Willd.) seeds. Bioscience Biotechnology and Biochemistry, Tokyo, v. 68, n. 1, p. 231-234, 2004.

MASCARENHAS, M. H. T.; RESENDE, L. M. A. Cadeia produtiva da batata no estado de Minas Gerais. In Seminário mineiro de bataticultura, 4, 2003. Poços de caldas. Empresa de pesquisa agropecuária de Minas Gerais, 2003. P. 31-54.

MORETTO, E. FETT R.; GONZAGA, L.V.; KUSKOSKI, E.M. Introdução à ciência de alimentos. Editora da UFSC, 255p, 2002.

OLIVEIRA, A.P.; GONDIM, P. C.; SILVA, O. P. R.; OLIVEIRA, A. N. P.; GONDIM, S. C.; SILVA, J. A. Produção e teor de amido de batata-doce em cultivo sob adubação com matéria orgânica. Revista Brasileira de Engenharia Agrícola e Ambiental, v17, n. 8, p.830-834, 2013. 
PIERARSKI, F. V. B. W. Folha de abóbora: caracterização físico-química, mineral e efeito da adição na reologia da massa e na qualidade sensorial de pães contendo fibra alimentar. 2009. 147f. Mestrado (Tecnologia em Alimentos)

Universidade Federal do Paraná, Curitiba.

SILVA, R. G. V. Caracterização físico-quimica de farinha de batata-doce para produtos de panificação. Dissertação, Bahia, 2010.

SILVA, R.G.V.; Caracterização físico-química de farinha de batata-doce para produtos de panificação. Dissertação de mestrado em engenharia de alimentosUniversidade Estadual do Sudoeste da Bahia, Bahia, 2010.

SIMON, Aline. Elaboração de Brownie de chocolate sem glúten com a utilização de farinha de arroz e trigo sarraceno. Trabalho de conclusão de curso em engenharia de alimentos - Universidade Federal do Rio Grande do Sul, 2014.

SOARES, I.; BASTOS, E. G. P.; SOBRINHO, T. J. S.; ALVIM, T. C.; SILVEIRA, M. A.; AGUIAR, R. W. S.; ASCÊNCIO, S. D. Conteúdo fenólico e atividade antioxidante de diferentes cultivares de Ipomoea batatas (L.) Lam. obtidas por melhoramento genético para produção industrial de etanol. Revista de Ciências Farmacêuticas Básica e Aplicada, 2014; 35(3): 479-488.

ZUANY, M. G. P. Farinha de Inhame. Disponível em: $<$ http:// vivendoeaprendendo.blogs-pot.com/2007_11_18_archive.html-66k $>$. Acesso em: 10 set 2016.

\section{AGRADECIMENTOS}

Agradecimento ao LEAAL, Departamento de Nutrição - UFPE, pelo apoio na realização da composição centesimal da farinha experimental. 